\title{
Visual Hallucinations in Progressive Supranuclear Palsy
}

\author{
Spiridon Papapetropoulos Deborah C. Mash \\ Department of Neurology, University of Miami, Miller School of Medicine, Miami, Fla., USA
}

Dear Sir,

Progressive supranuclear palsy (PSP) is the most common and best recognized atypical parkinsonian syndrome. The clinical characteristics of PSP as described by Steele, Richardson and Olszewsky [1] in 1964 include parkinsonian features, postural instability, vertical supranuclear gaze palsy, pseudobulbar symptoms and cognitive decline. However, it has been widely recognized that PSP is characterized by an ever-expanding and 'diverse' clinical phenotype [2] that among others includes a florid neuropsychiatric symptomatology [3]. This report focuses on the description of hallucinations in a small cohort of clinically and pathologically diagnosed PSP patients.

Our study population consisted of 22 consecutive PSP patients. All subjects consented during life to donate their brains to the University of Miami Brain Endowment Bank $^{\mathrm{TM}}$. Brain donors were recruited and registered with the Brain Bank through a campaign performed throughout the United States over the last decade. All donors completed the Brain Bank's PD registry form (a 128-item, self-administered questionnaire) providing information about demographics, environmental exposures, personal and family history, activities of daily living, clinical and treatment details. Yearly updates on all brain donors were obtained until death. Medical and hospital records were collected on an annual basis and all pertinent information was entered into a database. For patients with cognitive impairment a caregiver with knowledge of the patient's family and personal medical history completed the registry form. Upon death, remaining medical records were collected and neuropathological analyses were performed. Patients were brought to autopsy according to standardized protocols for brain handling and processing.

The clinical diagnosis of PSP was made either by movement disorders specialists and/or general neurologists before death and was clearly documented in the medical files. Two patients were only evaluated by a general neurologist. Multiple documented neurological evaluations throughout the course of the patients' illness were reviewed. Diagnoses were re-confirmed after death by a movement disorders specialist (S.P.), who applied standard criteria for PSP [4] (vertical supranuclear gaze palsy, prominent postural instability, falls in the first year of onset, and no evidence of other disorders to explain the neurologic findings). Diagnoses of dementia and psychiatric disorders were made by the patients' treating physicians and were clearly documented in the medical files. Clear documentation of dementia included diagnosis by a psychiatrist, formal psychometric testing and/or Mini-Mental State Examination $<24$ [5]. The character- istics of hallucinations were extracted from registry forms and medical notes.

All brains were formalin-fixed and sectioned according to standard protocols [6]. Neuropathologic diagnosis of PSP was based on well-accepted criteria, using conventional staining techniques [7]. The study was approved by the local institutional review board.

Among 22 patients [15 (68.2\%) men and $7(37.8 \%)$ women] with PSP [mean age at onset 67.0 years (SD 9.5), at death 72.9 years (SD 8.4) and disease duration from symptom onset to death 5.5 years (SD 2.5)], $2(9.1 \%)$ experienced visual hallucinations during the course of their disease. One of the two patients also complained of auditory hallucinations. Two factors of potential importance should be considered about our cohort: $11(50 \%)$ had a disease duration of more than 4 years and $16(72.7 \%)$ were on $L$-dopa for more than 5 years.

The neuropathological findings in both cases with hallucinations were typical of PSP: case 1 had no significant neocortical or hippocampal involvement and no subcortical white matter lesions. Case 2 demonstrated enough pathologic features to satisfy the CERAD diagnostic criteria for probable Alzheimer's disease [6]. Both cases demonstrated extensive substantia nigral gliosis and neuronal loss, with extraneuronal neuromelanin and neurofibrillary

\section{KARGER}

(c) 2005 S. Karger AG, Basel

Fax +4161306 1234 E-Mail karger@karger.ch www.karger.com www.karger.com/ene
Spiridon Papapetropoulos, MD, $\mathrm{PhD}$

Department of Neurology

University of Miami, School of Medicine, Room 4004

1501 NW 9th Avenue, Miami, FL 33136 (USA)

Tel. +1 305243 8461, Fax +1 305243 3649, E-Mail spapapetropoulos@med.miami.edu 
tangles (NFTs). NFTs were also seen in thalamus and other basoganglionic structures in both cases. A detailed description of the characteristics of our PSP cohort is provided elsewhere [2].

Case 1: A right-handed, Caucasian female was diagnosed with parkinsonism at the age of 69 when she noted a progressive lack of coordination of her left upper limb followed (a few months later) by postural instability and falls. The patient was subsequently started on $L$-dopa with moderate response. She eventually (over a period of 2 years) developed all of the characteristic features of PSP including vertical gaze palsy, marked axial rigidity, bradykinesia, and pseudobulbar symptomatology with swallowing difficulties. About 5 years into her disease and while on low doses of $L$-dopa (300 mg/day) and selegiline (10 mg/day) she started having vivid nightmares which she had difficulty discerning from reality. About 1 year later and while her cognitive function was moderately affected, she started experiencing visual hallucinations. For a period of about 1 year they remained benign. She would constantly see the same image of her son's dog. The image was present for a few seconds and then would suddenly vanish. Subsequently, her hallucinations became more frequent, prolonged, complex and frightening and she also started experiencing auditory hallucinations (threatening voices). She was placed on risperidone $(0.5 \mathrm{mg} /$ day $)$ with moderate improvement in her symptoms. However, after an exacerbation of her parkinsonian symptoms (mainly rigidity) this was gradually replaced by clozapine (up to $50 \mathrm{mg} /$ day), which failed to produce the same effect. About 7 years into her disease $L$-dopa was discontinued because of loss of function. However, neither the intensity nor the characteristics of the hallucinations were affected by the discontinuation of $L$-dopa. The patient's psychotic symptomatology responded mildly to a combination of clozapine and bupropion hydrochloride. She died due to complications of PSP 1 year later.

Case 2: A right-handed, Caucasian male was diagnosed with parkinsonism at the age 59 after he developed an atypical (6- to 8$\mathrm{Hz}$ ) resting tremor of his right hand. He was gradually started on $L$-dopa (400 mg/day), with mild (about $40 \%$ ) improvement. His condition deteriorated over the next few years with marked axial rigidity and bradykinesia. About 5 years into his disease the patient was diagnosed with PSP after developing a rather typical symptomatology, in- cluding a disturbance of vertical gaze, an increasing disturbance in balance, swallowing difficulties and moderate cognitive impairment. A trial of amantadine and an increase in the $L$-dopa dose (up to $800 \mathrm{mg}$ / day) failed to provide further symptomatic relief. There was considerable sleep disturbance with fragmentation and daytime sleepiness, partly treated with zolpidem tartrate. At the same time, while under $L$-dopa (400 mg/day) and about 5.5 years into his disease the patient started experiencing benign visual hallucinations. They consisted of 'friendly' fragmented faces that appeared in dim surroundings. They lasted for a few minutes and then disappeared. His medical history, review of systems and neuroimaging at the time did not indicate any triggering factors. There was no therapeutic intervention for the hallucinatory symptoms since they remained benign and spontaneously remitted about 1 year later, never to reappear. The patient died a few years later of aspiration pneumonia.

We report here our findings on the characteristics of hallucinations from a small cohort of PSP patients. Only 2 out of 22 PSP patients $(9.1 \%)$ reported visual hallucinations. Case 1 had auditory in addition to visual hallucinations. The hallucinatory experience was somewhat similar to that described by Parkinson's disease (PD) patients (for review see [8]). Symptoms were a late complication of the disease, developing several years after onset. Both patients were on long-term dopaminergic replacement therapy although there was no clear relation between symptom development and treatment parameters. Sleep abnormalities (vivid dreaming and fragmentation, respectively) preceded and significant $\operatorname{cog}$ nitive impairment accompanied the development of the hallucinations, in accordance with the continuum theory [9]. The symptoms improved with antipsychotic therapy in one patient that tried it. However, both cases presented here had additional atypical features: in case 1 the hallucinations did not subside after the discontinuation of $L$-dopa and in case 2 the symptoms remitted spontaneously, without discontinuation of therapy.

Hallucinations (mainly visual) are particularly common in PD and are present in about $20-40 \%$ of patients [10]. They are usually drug-related, although other factors may be important (i.e. cognitive impairment, disease severity and duration) $[10$, 11]. They have a major impact on the natural history of PD and have been identified as one of the cardinal risk factors for nursing home placement [12]. Hallucinations have been less frequently reported in PSP. A study comparing neuropsychiatric features of PD versus PSP patients found that hallucinations were reported in only 1 of the 27 PSP patients who were treated with dopaminergic agents, and concluded that regional neurochemical differences in cholinergic and monoaminergic degeneration may provide possible explanations for the finding of more frequent hallucinations in PD than in PSP patients [3]. In our cohort 2 out of 11 patients (18.2\%) that received chronic treatment with dopaminergic agents developed hallucinations.

Similarities in the characteristics of hallucinations described in PD and PSP suggest the involvement of similar mechanisms (denervation supersensitivity of mesocortical and mesolimbic dopaminergic receptors and other neurotransmitter imbalances [8]) in their development. Factors that may account for the differences in the frequency of visual hallucinations between PSP and PD patients (our previously reported frequency of hallucinations in PD patients of our Brain Bank cohort is 52.2\% [13]) are: (a) regional neurochemical differences in cholinergic and monoaminergic degeneration; (b) the decreased survival of PSP versus PD patients and (c) the early discontinuation of dopaminergic treatment due to loss of efficacy. Our report has certain limitations commonly seen in retrospective studies. The possibility of incomplete symptom recording as well as ascertainment and small sample bias should be considered. Larger prospective studies on the characteristics and prevalence of hallucinations in PSP are warranted.

\section{Acknowledgments}

The Brain Endowment Bank ${ }^{\mathrm{TM}}$ is funded in part by the National Parkinson Foundation (NPF). This work was supported in part by a grant from the National Parkinson Foundation (NPF 662891).

\section{References}

1 Steele JC, Richardson JC, Olszewski J: Progressive supranuclear palsy. A heterogeneous degeneration involving the brain stem, basal ganglia and cerebellum with vertical gaze and pseudobulbar palsy, nuchal dystonia and dementia. Arch Neurol 1964; 10:333-359.

2 Papapetropoulos S, Gonzalez J, Mash DC: Natural history of progressive supranuclear palsy. A clinicopathologic study from a population of brain donors. Eur Neurol 2005;54: $1-9$ 
3 Aarsland D, Litvan I, Larsen JP: Neuropsychiatric symptoms of patients with progressive supranuclear palsy and Parkinson's disease. J Neuropsychiatry Clin Neurosci 2001;13:4249.

4 Litvan I, Agid Y, Calne D, Campbell G, Dubois B, Duvoisin RC, Goetz CG, Golbe LI, Grafman J, Growdon JH, Hallett M, Jankovic J, Quinn NP, Tolosa E, Zee DS: Clinical research criteria for the diagnosis of progressive supranuclear palsy (Steele-Richardson-Olszewski syndrome): report of the NINDS-SPSP international workshop. Neurology 1996;47: $1-9$.

5 Folstein MF, Folstein SE, McHugh PR: 'Minimental state'. A practical method for grading the cognitive state of patients for the clinician. J Psychiatr Res 1975;12:189-198.
6 Mirra SS, Heyman A, McKeel D, Sumi SM, Crain BJ, Brownlee LM, Vogel FS, Hughes JP, van Belle G, Berg L: The Consortium to Establish a Registry for Alzheimer's Disease (CERAD). 2. Standardization of the neuropathologic assessment of Alzheimer's disease. Neurology 1991;41:479-486.

7 Litvan I, Agid Y, Jankovic J, Goetz C, Brandel JP, Lai EC, Wenning G, D'Olhaberriague L, Verny M, Chaudhuri KR, McKee A, Jellinger K, Bartko JJ, Mangone CA, Pearce RK: Accuracy of clinical criteria for the diagnosis of progressive supranuclear palsy (Steele-Richardson-Olszewski syndrome). Neurology 1996;46: 922-930.

8 Papapetropoulos S, Mash DC: Psychotic symptoms in Parkinson's disease. From description to etiology. J Neurol 2005;252:753-764.

9 Moskovitz C, Moses H 3rd, Klawans HL: Levodopa-induced psychosis: a kindling phenomenon. Am J Psychiatry 1978;135:669675.
10 Papapetropoulos S, Argyriou AA, Ellul J: Factors associated with drug-induced visual hallucinations in Parkinson's disease. J Neurol 2005;252:1223-1228.

11 Fenelon G, Mahieux F, Huon R, Ziegler M: Hallucinations in Parkinson's disease: prevalence, phenomenology and risk factors. Brain 2000; $123: 733-745$.

12 Goetz CG, Stebbins GT: Risk factors for nursing home placement in advanced Parkinson's disease. Neurology 1993;43:2227-2229.

13 Papapetropoulos S, Gonzalez J, Lieberman A, Villar JM, Mash DC: Dementia in Parkinson's disease: a post-mortem study in a population of brain donors. Int J Geriatr Psychiatry 2005; 20:418-422. 